\title{
Erratum to: Toward a geometrical foundation for physics
}

\author{
Sharon Eitan
}

Published online: 25 February 2015

(C) Chapman University 2015

\section{Erratum to: Quantum Stud.: Math. Found. DOI 10.1007/s40509-014-0022-6}

I correct a small error in the new formulation of the Dirac equation presented in my paper, and revise an argument I presented regarding the relation between the deterministic nature of EMO dynamics and quantum non-determinism.

\section{Correction (1)}

In the mass term of the new formulation of the Dirac equation suggested in my paper I mistakenly omitted a factor of magnitude 1 and units $\frac{1}{\text { length }}$ required by unit considerations. This omission occurred consistently in all the appearances of the new formulation in the text (with momentum in the $\hat{z}$ direction, momentum in the $\hat{y}$ direction, etc).

For example:

$$
\frac{\partial \psi_{1}}{\partial \text { time }}=-i \psi_{1}-\frac{\partial \psi_{3}}{\partial z} \hat{z}
$$

Should be replaced by

$$
\frac{\partial \psi_{1}}{\partial \text { time }}=-i a \psi_{1}-\frac{\partial \psi_{3}}{\partial z} \hat{z}
$$

where $a=1$ has units $\frac{1}{\text { length }}$

And so on.

The online version of the original article can be found under doi:10.1007/s40509-014-0022-6.

S. Eitan $(\bowtie)$

Tel Aviv, Israel

e-mail: sharoneitanphysics@gmail.com 


\section{Correction (2)}

I briefly discussed the question of the relation between the determinism of EMO dynamics and the non-determinism of quantum physics, and proposed that even if we were given complete initial conditions for EMO space at some value of $t$, the resulting physical theory would be non-deterministic. Following remarks by Guy Moshel and Adiel Meyer, I am no longer sure of that. However, I feel that by focusing on the question of what might happen if we were given complete initial conditions for EMO space, I obscured a more important point, namely, that according to EMO theory, it is impossible to obtain initial conditions for a given value of $t$ via physical measurement. Given a value of $t$, it is impossible to stipulate that our measurements yield results pertaining to the specific given value of $t$, because the results of any physical measurement depend on the state of observed EMO combinations during all the values of $t$ at which type (ii) interactions occur between the observer and observed, which may or may not include the given value of $t$.

Let us think of the momentary EMO space positions of EMO focal points as representing positions in $S^{3}$, in accordance with what I called "the hard approach" to the question of the relation between EMO space and $S^{3}$. I speculated that the density of $S^{3}$ points represented in EMO space might turn out to be bounded by the demands of EMO combination stability, and suggested that the existence of such a bound may play a part in an EMO theory of gravity. Attempting to make physical predictions based on such a picture, one can expect to initially have to take into account the entire range of possibilities for the number of EMOs in EMO space, going to infinity, and then, for the sake of the gravitational discussion, observe a correlation between a bound on the number of EMOs and EMO combination stability. Each possibility for the number of EMOs would of course correspond to a wide range of possible initial conditions, determining the positions of EMO focal points and orientations of EMO planes. 